\title{
The iceberg tip and the rest. Mental health care for people with schizophrenia in five European centres
}

\author{
THOMAS BECKER, LUIS GAITE, MARTIN KNAPP, HELLE CHARLOTTE KNUDSEN, \\ MORVEN LEESE, MIRELLA RUGGERI, AART SCHENE, MICHELE TANSELLA, \\ GRAHAM THORNICROFT, JOSÉ LUIS VÁZQUEZ-BARQUERO, BIRGITTE WELCHER, \\ BOB VAN WIJNGAARDEN
}

The 'iceberg' analogy was used by Rose (1993) to describe the limitations, in preventive psychiatry, of concentrating on high-risk groups only. As symptom levels are distributed continuously in populations prevalence rates are likely to be best reduced by affecting overall symptom levels in the whole population. Similarly, when the aim is to get rid of an iceberg to prevent ships from sinking heating the sea water to melt down all its parts would be more effective than shooting off its visible portions. Mental health care, in its complexity, could be compared to an iceberg, and a cross-sectional analysis such as the EPSILON study provides limited glimpses at a multi-faceted reality most of which remains submerged. This has been acknowledged in a recent set of papers which emphasised the shortcomings of research methodology in describing psychiatric services across countries (Beecham \& Munizza, 2000). The design of the EPSILON study, a cross-sectional investigation of care for people with schizophrenia in five urban European centres (Amsterdam, Copenhagen, London, Santander, Verona) is described in an accompanying editorial (Thornicroft et al., 2002). The study was aimed at instrument adaptation and service comparison across centres. What, then, were the results of the EPSILON study?

Indirizzo per la corrispondenza: Professor T. Becker, Department of Psychiatry, University of Leipzig, Liebigstrasse 22B, D-04105 Leipzig (Germany).

Fax: +49-341-972.4409

E-mail: beckt@medizin.uni-leipzig.de

\section{NEEDS}

The Camberwell Assessment of Need (CAN-EU; McCrone et al., 2000) was used to assess needs for care:

- The mean number of needs, across the centres, varied from just under five (Santander 4.8 [95\% CI 4.2-5.4], Verona $4.9[4.3-5.5]$ ) to about six (Amsterdam 6.3 [5.57.1], London 6.0 [5.4-6.6]).

- The numbers of met needs were between about three and four, and the mean number of unmet needs varied from a minimum of 1.3 in Copenhagen to a maximum of 2.5 in Amsterdam (McCrone et al., 2001).

- Differences between the sites were significant for unmet needs, both with and without adjustment for socio-demographic and illness-related covariates.

- Service supply effects appeared moderate although the inspection of data suggested some effects of differences in provision, e.g. where day care was concerned.

- The gap between Copenhagen and the other sites widened after adjusting for patient characteristics, suggesting that the low number of unmet needs in that site could not be attributed to sample differences.

- Relatively high unmet needs scores in Amsterdam and London could be due to expectations being higher or because of relative service inefficiencies.

Accommodation was rated as 'not a problem' most frequently in Santander followed by Verona. This could be due to a cultural effect (the family perceiving a duty to look after the patient), but it could also reflect limited service provision of supported accommodation (with the consequence of non-identification of need due to under-provision), or both. Daytime activities were often not perceived as a need in Verona, but in Santander they we- 
re frequently reported as an unmet need. In Santander the use of day care was minimal while it was high (44\%) in Verona (with only $31 \%$ of Verona patients reporting a need for daytime activities) which might suggest overprovision in the latter and under-provision in the former site (McCrone et al., 2001). There was a high proportion (four-fifths), in London, of patients reporting a met need regarding psychotic symptoms. This differed from Verona (57\%) and could reflect differences in attitudes towards and relative emphasis placed on antipsychotic medication (in the UK and Italy). The high rate of met psychological distress needs observed in Verona (which contrasted with a high proportion of unmet needs in Amsterdam) could be a related finding reflecting differential psychological focus of care models in the two sites. Some of the needs items could reflect problem areas of large urban agglomerations, with unmet needs in company, intimate relationships, sexual expression and daytime activity in Amsterdam, and unmet needs in 'practical living' domains in London (McCrone et al., 2001).

\section{FAMILY CAREGIVING}

The Involvement Evaluation Questionnaire (IEQ-EU) was used to assess caregiver involvement (van Wijngaarden et al., 2000):

- The factors derived from simultaneous components analysis were comparable with the original IEQ factors with minor deviations (van Wijngaarden et al., submitted for publication).

- There were four factors with comparable reliability (tension, supervision, worrying, and urging).

- The rank order of mean scores was identical across the sites, and worrying had the highest mean scores.

- In terms of the level of caregiving consequences the five sites differed significantly on all scales, with the highest scores generally found in Verona, and the lowest in Amsterdam and Copenhagen.

- In all cases the Verona scores were about twice as high as the Amsterdam and Copenhagen scores, with Santander and London holding an intermediate position.

- IEQ scores were generally higher if patients were older, were native speakers, had more contact with the caregiver, had a caregiver with impaired coping, or lived in places with fewer psychiatric beds.

There were differences in sample characteristics between the sites, and adjusted IEQ scores were computed and resulted in the same overall picture with Verona, Santander and London scores being higher than tho- se in Amsterdam and Copenhagen. The scale scores were adjusted for the important variables. Therefore, the findings were not likely due to sample differences (as variables known from previous research to predict IEQ scores were entered in the analysis of covariance).

Where 'real' differences in caregiving are concerned the number of residential care places (which has a substantial effect on informal caregiver involvement) did not go all the way to explain inter-site differences. However, it needs to be borne in mind that the study failed to fully account for differences in the quality of local mental health care provision. Where the 'cultural' explanation is concerned cross-site differences resembled those reported by Magliano et al. (1998). In terms of institutional vs. family residential arrangements, and quantitative involvement of informal caregivers there were some inter-site differences which fit into this pattern of more intense family caregiving in southern European sites. However, when IEQ scores were adjusted for sample differences the clear north-south divide had disappeared.

\section{SERVICE SATISFACTION}

Patient satisfaction was measured using the Verona Service Satisfaction Scale (VSSS-EU; Ruggeri et al., 2000):

- In terms of satisfaction domains Verona had the smallest proportion of patients who were unsatisfied with service efficacy, type of intervention and relative's involvement; Amsterdam and Verona had the smallest share of unsatisfied service users with respect to information, Santander and Copenhagen had the smallest proportion of unsatisfied patients where professionals' skills and behaviour were concerned, and in Copenhagen the fewest unsatisfied service users were found with respect to service access.

- Patient satisfaction differed significantly across the sites with scores ranging mostly from 3 to 4 on a 5-point Likert scale (' 1 ' being 'terrible', and ' 5 ' being 'excellent').

- The highest scores were observed in Copenhagen, the lowest in London (overall satisfaction, professionals' skills and behaviour, self-perceived efficacy).

- Also, satisfaction with access to services was highest in Copenhagen (lowest in Amsterdam and Verona).

- Verona had the highest satisfaction score concerning the types of intervention available (Santander scoring lowest).

- After adjusting for differences in sample characteristics the differences remained significant, and the gap 
between Copenhagen and the other sites widened.

- Considering specific areas of dissatisfaction, subjects were generally least satisfied in London and most satisfied in Copenhagen (with other sites scoring both highest and lowest on individual satisfaction domains), and a wide variability of scores was observed.

- In stepwise multiple linear regression analysis, living in London and Santander (centre effect), being retired or unemployed, having a high number of hospital admissions, a high level of psychopathological impairment, a large number of unmet needs and poor quality of life (in the domains of social relations and health) correlated with lower service satisfaction (Ruggeri et al., submitted for publication).

The high degree of variability of individual VSSS-EU item scores across the five sites and the absence of any specific pattern of differences between north and south European sites suggest that cross-cultural differences cannot be summarised as a north-south divide, and service specificity of satisfaction scores is suggested by the data. It is noteworthy that 'information about illness' and 'involvement of relatives' are the satisfaction domains with the worst performance in all the sites. These findings are in line with other studies (Boardman et al., 1999; Leese et al., 1998; Perreault et al., 1999), and the issues of information and relative involvement require specific attention in service planning and quality management. Among the predictors of service satisfaction the social relations and health domains of the quality of life (QoL) measure had the strongest impact.

\section{SUBJECTIVE QUALITY OF LIFE}

The Lancashire Quality of Life Profile (LQOLP-EU; Gaite et al., 2000) was used to assess QoL:

- Where subjective QoL is concerned the average mean LQOLP score, at 4.67 (SD 0.76) was close to the midpoint of 4 (towards the better end, '7' being 'completely satisfied'; Gaite et al., in press).

- Satisfaction scores were lowest in the domains of work and finances.

- Copenhagen was the centre with the highest ratings in six of the nine QoL domains (work, leisure activities, religion, finances, living situation, safety) while London was the centre scoring lowest.

- Among the predictors of subjective QoL scores the BPRS anxiety-depression and positive symptom subscales, number of psychiatric admissions and history of alcohol abuse accounted for $18 \%$ of the variance.

- Objective QoL aspects (being in Copenhagen [po- sitive], London or Santander [negative], having a reliable friend, daily contact with family, number of leisure activities, age and work) accounted for about $19 \%$, and the final model including clinical and sociodemographic variables (BPRS anxiety-depression score, being in Copenhagen or London, having a reliable friend, daily contact with family, BPRS positive symptoms) explained $29 \%$ of the variance of subjective QoL.

\section{Gender}

Male patients had higher negative symptom scores on the BPRS. They had more needs in the areas of harm to others and substance misuse, but there was no evidence of gender differences with regard to needs for accommodation, psychotic symptoms, or for sexual expression (Thornicroft et al., in press). Women had more needs for childcare, and in the area of self-harm. Men and women showed similar levels of both overall objective and subjective QoL, but there were differences in the domains of family life (men higher) and leisure (women higher). Associations between those characteristics in the BPRS, CAN-EU and LQOLP-EU that did differ between men and women were further analysed using graphical models. This analysis showed that the models were rather similar for men and women (importance of 'leisure' QoL subscore and some of its associations, association between symptom severity and self-harm) but not in all respects ('leisure' QoL directly and negatively associated with 'self-harm' in women but with 'harm to others' in men). No differences were found for satisfaction with services (total score and subscores), nor in caregiver burden, even when the gender of the caregiver as well as the patient was taken into account. Overall there were few differences in service use, and those that were present were site-specific: inpatient service use varied between men and women, with a different pattern for Copenhagen (women higher) and London (men higher). In Amsterdam, use of day care was higher for men than for women. The overall picture suggests that men and women were rather similar with regard to the domains assessed in the study, and seem to match men and women in the general population. Service use differences might be determined by external factors. The conclusion is that, if anything, services should make provision more alike for men and women, not more different, except in some few very obvious areas (with the caveat that perhaps the right questions were not asked).

\section{'Ways of life'}

It is of crucial importance in understanding a person's mental health and mental health care provision to know

Epidemiologia e Psichiatria Sociale, 11, 1, 2002 
in which country, city or region he or she lives. In the EPSILON study, the cities in which centres were located varied in size with London being largest, and Amsterdam being larger than Copenhagen, Santander and Verona. London had the oldest patient population which had been in contact with mental health services for the longest period of time. Almost three-quarters of patients in Santander and half in Verona lived with relatives, and these two sites had the highest percentages of people living in mainstream accommodation. On the other hand, between two thirds and almost half of the patients in Copenhagen, Amsterdam and London lived alone. There was more shared living with mother or father in Santander, and sharing a household was more frequent. Informal caregivers in Verona were less used to the fact of their relative being mentally ill, and they were less able to cope with this fact when compared to caregivers in other sites.

There were other objective QoL differences across the sites. Thus, employment rates were highest in Copenhagen (35.3\%) and lowest in London (8.3\%). On the other hand, London patients reported their participation in most leisure time activities while Verona patients participated least. There were differences in the strength of religious beliefs (Verona, Santander and Copenhagen - high, London and Amsterdam - low). With regard to state benefits only $35.5 \%$ of the Verona patients received such benefits while $100 \%$ received benefits in Copenhagen. In terms of mental health care the majority of patients received psychopharmacological treatment in all the study sites. In London psychotherapy and rehabilitation services were provided to the lowest proportion of patients. In Santander social interventions were provided to the smallest number of subjects, and the highest level of under-provision of care was reported. Amsterdam, Copenhagen and Verona tended to provide the full spectrum of interventions. The rate of contact with somatic medical care was high in Copenhagen and Amsterdam, and low in Santander and Verona.

In describing the pattern of differences Copenhagen can be described as a relatively affluent and safe place with comprehensive service provision while Santander has a more restricted range of services. Living with family, in the southern sites, is contrasted with living alone in the northern European centres, and London is characterised by some degree of social deprivation among people suffering from schizophrenia. Is there any indication, then, that these generic differences bear relevance on the EPSILON study results? "If one thing is better in one place this need not apply to all things, and culture colours perception".
The directions and extremes of inter-site differences vary in this study. On many, albeit not all, of the domains taken into consideration the centres in Copenhagen and London represent opposite end points in the distribution of data. Looking in detail, Copenhagen is considered a safe city with a small proportion of ethnic minorities, and it has the highest numbers of patients employed, having formal friendships, receiving state benefits, holding religious beliefs, and being involved in leisure activities. In London there is a less strong presence of religious beliefs and higher percentage of non-white European population. There is also a higher percentage of patients unemployed or who are victims of violence. A clear concordance between subjective $\mathrm{QoL}$ and objective living conditions was found in some instances (satisfaction with work greater in Copenhagen and Verona where the lowest number of unemployed were found, satisfaction with safety higher in Copenhagen, Santander and Verona, centres with a lower number of victims of violence, satisfaction with family and social relations higher in Santander with frequency of daily contact with relatives close to $96 \%$ ).

However, there were also cases in which the objective and subjective perspective were not concordant. In the case of finances more than $90 \%$ of patients received social benefits in Copenhagen but the specific level of satisfaction in this area was lower than in Santander and Verona where the number of persons receiving social benefits was considerably lower. Thus, the perception of reality appears to be different across the sites. It might be that in the southern European centres the family constitutes an important source of support and income which might account for the objective-subjective discrepancy. Culture could influence the perception of objective indicators and have an impact on subjective QoL (and on the experience of informal caregivers, too). In the domains of 'living situation' and 'family relations' the background was that of a higher percentage of patients living with family in Santander and Verona. This cannot be accounted for by differences in service systems as Verona has developed a network of community services comparable to those in Amsterdam, Copenhagen and London. However, Copenhagen patients also had high satisfaction with their way of life although most lived alone. In Copenhagen, service availability might contribute to satisfaction, and the general perspective on the preferred ways of living could be somewhat different.

Services in Copenhagen received the highest level of funding, i.e. the costs of care were highest (see Chisholm \& Knapp, 2002). This difference between Copenhagen and the other sites can be traced throughout most of the 
areas assessed whether we consider the range and number of services, staffing levels or provision of residential care places. This may make for the decisive difference that differentiates Copenhagen from the other sites: here was a well-resourced service with reasonable staffing providing care for people comparatively severely ill (as adjustment procedures showed).

\section{The 'centre effect'}

Returning to the iceberg analogy this study has looked at the care processes, all to be envisaged as icebergs of complex shape, in five European centres from a variety of angles. A caveat must be considered: Extrapolations from the centres in which the study was conducted to the respective countries must be avoided ("one iceberg does not tell you much about the surrounding icebergs which may, indeed, be different"). The five centres studied cannot be considered representative of the five countries, and comparative research at the country level, with the amount of detailed information collected in the EPSILON study is a very difficult (or impossible?) undertaking.

Also, with a cross-sectional design and the outcome domains studied being closely associated with and overlapping each other there is some element of circularity in the argument ("no matter whether you look at shape or diameter or degree of immersion: it is all related to the fact of icebergs swimming in the water"), and there is certainly scope for research on broad and common 'underlying factors' determining inter-site homogeneity and variation. In this study, the data on complex living situations of people with severe mental illness showed many aspects in common, and similar types of service are provided ("don't these icebergs look alike most of the time?"). On the other hand, predictive models developed in the EPSILON study suggest that the variable 'centre' which includes characteristics of the place of residence, the cultural setting and local provision of services has a substantial influence on the expectations of people with schizophrenia and on the perception of their individual satisfaction with life (Gaite $e$ t al., in press). If we want to know more about what makes good mental health care we need to start looking into the care processes in much more detail. For the time being we can say that 'places feel different' ("somehow, when you look at them carefully each one of these icebergs is unique") but the real shape of the various icebergs is still under scrutiny. More detailed service description from the EPSILON study is forthcoming (Becker et al. in preparation), and we believe that if the type of research approach discussed here is accompanied by researchers and planners vi- siting each others' services (and places) then mental health care planning could be based on a broader empirical base and on the narratives of psychiatric reform in the different centres, regions, countries and cultures.

\section{REFERENCES}

Beecham J. \& Munizza C. (2000). Introduction: assessing mental health in Europe. Acta Psychiatrica Scandinavica 102, Suppl. no. 405, 5 7.

Boardman A.P., Hodgson R.E., Lewis M. \& Allen K. (1999). North Staffordshire Community Beds Study: longitudinal evaluation of in-patient units attached to community mental health centres. I. Methods, outcome and patients satisfaction. British Journal of Psychiatry 175, 70-78.

Chisholm D. \& Knapp M. (2002). The economics of schizophrenia care in Europe: the EPSILON study. Epidemiologia e Psichiatria Sociale 11, 12-17.

Gaite L., Vázquez-Barquero J.L., Arriaga Arrizabalaga A., Schene A.H., Welcher B., Thornicroft G., Ruggeri M., Vázquez-Bourgon E., Pérez Retuerto M., Leese M. \& the EPSILON Study Group (2000). Quality of life in schizophrenia: development, reliability and internal consistency of the Lancashire Quality of Life Profile - European Version. British Journal of Psychiatry 177, Suppl. no. 39, 49-54.

Gaite L., Vázquez-Barquero J.L., Borra C., Ballesteros J., Schene A., Welcher B., Thornicroft G., Becker T., Ruggeri M., Herrán A. \& the EPSILON study group (in press). Acta Psychiatrica Scandinavica.

Leese M., Johnson S., Slade M., Parkman S., Kelly F., Phelan M. \& Thornicroft G. (1998). User perspective on needs and satisfaction with mental health services. British Journal of Psychiatry 173, 409415.

Magliano L., Fadden G., Madianos M., Caldas de Almeida J.M., Held T., Guarneri M., Marasco C., Tosini P. \& Maj M. (1998). Burden on the families of patients with schizophrenia: results of the BIOMED I study. Social Psychiatry and Psychiatric Epidemiology 33, 405-412.

McCrone P., Leese M., Thornicroft G., Schene A.H., Knudsen H.C., Vázquez-Barquero J.L., Lasalvia A., Padfield S., White I.R., Giffiths G. \& the EPSILON study group (2000). Reliabilty of the Camberwell Assessment of Need - European Version. British Journal of Psychiatry 177, Suppl. no. 39, 34-39.

McCrone P., Leese M., Thornicroft G., Schene A.H., Knudsen H.C., Vázquez-Barquero J.L., Tansella M., Becker T. \& the EPSILON study group (2001). A comparison of needs of patients with schizophrenia in five European countries: the EPSILON Study. Acta Psychiatrica Scandinavica 103, 370-379.

Perreault M., Rogers W.L., Leichner P. \& Sabourin S. (1999). Patients' requests and satisfaction with services in an outpatient psychiatric setting. Psychiatric Services 47, 287-292.

Rose G. (1993). Editorial: Mental disorder and the strategies for prevention. Psychological Medicine 23, 553-555.

Ruggeri M., Lasalvia A., Dall'Agnola R., van Wjingaarden B., Knudsen H.C., Leese M., Gaite L., Tansella M. \& the EPSILON Study Group (2000). Development, internal consistency and reliability of the Verona Service Satisfaction Scale - European Version. British Journal of Psychiatry 177, Suppl. no. 39, 41-47.

Ruggeri M., Lasalvia A., Bisoffi G., Schene A., Knudsen H.C., Thor- 
nicroft G., Becker T., Knapp M., Gaite L. \& Tansella M. (submitted for publication). Satisfaction with mental health services among schizophrenic patients in five European countries: results from the EPSILON study.

Thornicroft G., Becker T., Knapp M., Knudsen H.C., Schene A., Tansella M., Vazquez-Barquero J.L. \& the EPSILON Study Group (2002). The conduct of successful multi-site EU collaborative research: the EPSILON Study. Epidemiologia e Psichiatria Sociale $11,2-5$.

Thornicroft G., Leese M., Tansella M., Howard L., Toulmin H., Herrán A. \& Schene A. (in press). Gender differences in living with schizophrenia. A cross-sectional European multi-site study. Schizophrenia Research. van Wijngaarden B., Schene A.H., Koeter M., Vázquez-Barquero J.L., Knudsen H.C., Lasalvia A., McCrone P. \& the EPSILON Study Group (2000). Caregiving in schizophrenia: development, internal consistency and reliability of the Involvement Evaluation Questionnaire - European Version. British Journal of Psychiatry 177 , Suppl. no. 39, 21-27.

van Wijngaarden B., Schene A., Koeter M., Becker T., Knudsen H.C., Tansella M., Thornicroft, G., Vázquez-Barquero J.L., Lasalvia A., Leese M. \& the EPSILON Study Group (submitted for publication). People with schizophrenia in five European countries: conceptual similarities and intercultural differences in family caregiving. 